\title{
www.czasopisma.pan.pl \\ Computer Simulation of the Solidification Process Including Air Gap Formation
}

\author{
T. Skrzypczak *, E. Węgrzyn-Skrzypczak, L. Sowa \\ Faculty of Mechanical Engineering and Computer Science, Czestochowa University of Technology, \\ Armii Krajowej 21, 42-201 Częstochowa, Poland \\ *Corresponding author. E-mail address: t.skrzypczak@imipkm.pcz.pl
}

Received 26.06.2017; accepted in revised form 03.08.2017

\begin{abstract}
The paper presents an approach of numerical modelling of alloy solidification in permanent mold and transient heat transport between the casting and the mold in two-dimensional space. The gap of time-dependent width called "air gap", filled with heat conducting gaseous medium is included in the model. The coefficient of thermal conductivity of the gas filling the space between the casting and the mold is small enough to introduce significant thermal resistance into the heat transport process. The mathematical model of heat transport is based on the partial differential equation of heat conduction written independently for the solidifying region and the mold. Appropriate solidification model based on the latent heat of solidification is also included in the mathematical description. These equations are supplemented by appropriate initial and boundary conditions. The formation process of air gap depends on the thermal deformations of the mold and the casting. The numerical model is based on the finite element method (FEM) with independent spatial discretization of interacting regions. It results in multi-mesh problem because the considered regions are disconnected.
\end{abstract}

Keywords: Solidification process, Computer simulation, Finite element method, Multi-mesh approach, Air gap

\section{Introduction}

Casting process takes into account various thermal phenomena such as heat transfer in the direction from the hot solidifying region to the cold mold and further to the ambient or releasing the latent heat of liquid-solid phase transformation. Presented work deals with solidification of bronze in the permanent mold made of cast iron. These two regions are initially in perfect contact because entire casting is in the liquid state. At this early stage of the process which takes place above liquidus temperature the liquid material adapts to the deforming shape of the mold. It changes with the start of solidification, when temperature drops enough to start liquid-solid phase transformation. From this moment the solid part of mold-casting system is subjected to thermal deformations. The mold expands due to temperature growth while the solid part of casting shrinks because of cooling process. These two phenomena often support one another and finally the air gap appears between the contact surfaces of the casting and the mold. It introduces significant thermal resistance at the mold-casting interface. Mathematical descriptions of such "non-ideal" thermal contact was described in [1]. Analytical solutions taking into account transient heat transfer through the gap was presented in [2]. Numerical solution of heat transport between two semi-infinite regions which uses the finite difference method (FDM) was discussed in [3]. Experimental investigations by measuring of air gap thickness for cylindrical and flat castings of aluminum alloys were presented in [4]. Nowadays the ideal thermal contact and contact via the gap are the area of interest of many scientists. The appropriate description of the mentioned phenomenon is required in the analysis of the solidification problems where the mold and the casting are considered $[5,6]$. Numerical investigations based on the finite element method (FEM) in the case of air gap formation process 
during solidification of pure metal was discussed in [7]. In the work [8] there was also the air gap observed during continuous casting process.

\section{Mathematical description}

Figure 1 shows the scheme of the problem. Entire region is divided into parts $\Omega_{M}$ and $\Omega_{C}$. The mold denoted by $\Omega_{M}$ is made of cast iron and filled with liquid bronze denoted by $\Omega_{C}$.

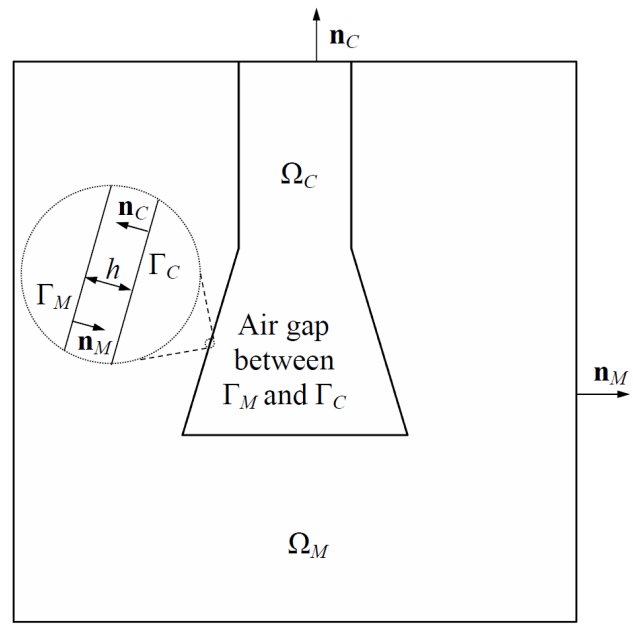

Fig. 1. Scheme of the problem

$\Gamma_{M}$ and $\Gamma_{C}$ are the boundaries of the mold and the casting respectively. The solidification process occurs in $\Omega_{C}$ and the heat is transported to the mold and to the ambient. Both the mold and the casting deform due to the changes of temperature. Thermal deformations lead to the air gap formation between the interacting parts of $\Gamma_{M}$ and $\Gamma_{C}$. At the bottom of the casting almost perfect thermal contact is introduced. The width of the gap $h$ varies according to time. Initially $\Gamma_{M}$ and $\Gamma_{C}$ are in perfect contact but the situation changes with the beginning of solidification.

The governing equations describing the solidification and heat transport are as follows:

$\frac{\partial}{\partial x}\left(\lambda_{C} \frac{\partial T^{C}}{\partial x}\right)+\frac{\partial}{\partial y}\left(\lambda_{C} \frac{\partial T^{C}}{\partial y}\right)=c^{*} \rho_{C} \frac{\partial T^{C}}{\partial t}$

$\frac{\partial}{\partial x}\left(\lambda_{M} \frac{\partial T^{M}}{\partial x}\right)+\frac{\partial}{\partial y}\left(\lambda_{M} \frac{\partial T^{M}}{\partial y}\right)=c_{M} \rho_{M} \frac{\partial T^{M}}{\partial t}$

where: $\lambda$ is the coefficient of thermal conductivity, $\rho$ - density, $c^{*}$ artificial heat capacity, $T$ - temperature, $c_{M}$ - heat capacity of the mold, $x, y$ - Cartesian coordinates, $t$ - time, while $M$ and $C$ indicate the mold and the casting.

According to the first solidification hypothesis [9] parameter $c^{*}$ is defined in the following way: $c^{*}=\left\{\begin{array}{cc}c_{s}, & T<T_{S} \\ 0.5\left(c_{s}+c_{l}\right)+\frac{L}{T_{L}-T_{S}}, & T_{S} \leq T \leq T_{L} \\ c_{l}, & T>T_{L}\end{array}\right\}$

where: $L$ - is the latent heat of solidification, $c_{s}, c_{l}$ - the specific heat of the solid $(s)$ and liquid $(l), T_{S}, T_{L}$ are the solidus and liquidus temperatures.

Above solidification model is simple and effective and is widely used, even to simulate the continuous casting process [10].

Equilibrium equations are the base to derive thermal deformations of the system:

$\frac{\partial \sigma_{x}^{(i)}}{\partial x}+\frac{\partial \tau_{x y}^{(i)}}{\partial y}=0, \quad \frac{\partial \tau_{y x}^{(i)}}{\partial x}+\frac{\partial \sigma_{y}^{(i)}}{\partial y}=0$

where: $\sigma_{x}, \sigma_{y}, \tau_{x y}, \tau_{y x}$ are the components of the symmetrical stress tensor $\left(\tau_{x y}=\tau_{y x}\right)$ and $(i)$ denotes the casting or the mold.

The components of stress tensor are as follows:

$\left[\begin{array}{l}\sigma_{x}^{(i)} \\ \sigma_{y}^{(i)} \\ \tau_{x y}^{(i)}\end{array}\right]=\frac{E_{(i)}}{1-v_{(i)}^{2}}\left[\begin{array}{ccc}1 & v_{(i)} & 0 \\ v_{(i)} & 1 & 0 \\ 0 & 0 & 0.5\left(1-v_{(i)}\right)\end{array}\right]\left[\begin{array}{c}\varepsilon_{x}^{(i)} \\ \varepsilon_{y}^{(i)} \\ \gamma_{x y}^{(i)}\end{array}\right]-\frac{E_{(i)} a_{(i)}}{1-v_{(i)}} T_{(i)}^{*}\left\{\begin{array}{l}1 \\ 1 \\ 0\end{array}\right\}$

where: $a$ is the coefficient of thermal expansion, $E$ - Young's modulus, $v$ - Poisson's ratio, $\varepsilon_{x}, \varepsilon_{y}, \gamma_{x y}$ - the components of strain tensor, $T_{(i)}^{*}=T^{(i)}-T_{r e f}, T_{r e f}$ - reference temperature.

The components of strain tensor are as follows:

$\varepsilon_{x}^{(i)}=\frac{\partial u_{x}^{(i)}}{\partial x}, \quad \varepsilon_{y}^{(i)}=\frac{\partial u_{y}^{(i)}}{\partial y}, \quad \gamma_{x y}^{(i)}=\frac{\partial u_{x}^{(i)}}{\partial y}+\frac{\partial u_{y}^{(i)}}{\partial x}$

where $u_{x}, u_{y}$ are the components of displacement vector.

Equations (5), (6) make possible to write equations (4) as the functions of unknown displacements $u_{x}, u_{y}$.

Presented mathematical model is supplemented by the appropriate initial and boundary conditions:

$T^{C}(t=0)=T_{0}^{C}, \quad T^{M}(t=0)=T_{0}^{M}$

$-\lambda_{C} \frac{\partial T^{C}}{\partial n_{C}}=\alpha_{C}\left(T^{C}-T_{a m b}^{C}\right), \quad-\lambda_{M} \frac{\partial T^{M}}{\partial n_{M}}=\alpha_{M}\left(T^{M}-T_{a m b}^{M}\right)$

$-\lambda_{M} \frac{\partial T^{M}}{\partial n_{M}}=\frac{\lambda_{g}}{h}\left(T^{M}-T^{C}\right)=\lambda_{C} \frac{\partial T^{C}}{\partial n_{C}}$

$u_{x}^{(i)}\left(x_{s}, y_{s}\right)=0, \quad u_{y}^{(i)}\left(x_{s}, y_{s}\right)=0$

where: $\alpha$ is the heat transfer coefficient, $T_{a m b}$ - ambient temperature, $n$ - direction of the vector normal to the external boundary, $\lambda_{g}$ - the coefficient of thermal conductivity in the gap, $x_{s}, y_{s}$ - the spatial coordinates of the immovable point of the casting and the mold. 


\section{Numerical model}

Governing equations of mathematical model are spatially discretized with the use of the Galerkin method. Then the implicit time integration scheme are adopted to obtain global FEM equations describing heat transfer in the system. The final form of these equations is shown below:

$$
\begin{aligned}
& \left(\mathbf{K}_{T M}+\frac{1}{\Delta t} \mathbf{M}_{T M}\right) \mathbf{T}_{M}^{f+1}=\mathbf{B}_{T M}+\frac{1}{\Delta t} \mathbf{M}_{T M} \mathbf{T}_{M}^{f} \\
& \left(\mathbf{K}_{T C}+\frac{1}{\Delta t} \mathbf{M}_{T C}\right) \mathbf{T}_{C}^{f+1}=\mathbf{B}_{T C}+\frac{1}{\Delta t} \mathbf{M}_{T C} \mathbf{T}_{C}^{f} \\
& \mathbf{K}_{D M} \mathbf{u}_{M}=\mathbf{B}_{D M} \\
& \mathbf{K}_{D C} \mathbf{u}_{C}=\mathbf{B}_{D C}
\end{aligned}
$$

where: $\mathbf{K}_{T M}, \mathbf{K}_{T C}$ are the global thermal conductivity matrices, $\mathbf{M}_{T M}, \mathbf{M}_{T C}$ - global heat capacity matrices, $\mathbf{B}_{T M}, \mathbf{B}_{T C}$ - right hand side vectors storing thermal boundary conditions, $\Delta t$ - time step, $f$ - time level, $\mathbf{K}_{D M}, \mathbf{K}_{D C}$ - global stiffness matrices, $\mathbf{B}_{D M}, \mathbf{B}_{D C}-$ vectors storing thermal loading.

In each time step the temperature distributions in the casting and the mold are obtained sequentially, then the thermal load is used to find the nodal displacements $\mathbf{u}_{C}, \mathbf{u}_{M}$. Obtained displacements are necessary to compute local width of the gap which determines thermal resistance between the mold and the casting. The procedure of incorporating the thermal contact boundary condition (9) into equations (11), (12) was discussed in [11].

\section{Example of calculation}

The in-home solver based on the finite element method was prepared to modeling described process. The computer simulation of the solidification process of the bronze casting in the permanent mold made of cast iron was made under the assumption that the mold and the casting are subjected to the thermal deformations. Temperature distribution and formation process of the air gap were investigated. The material properties used in the calculations are compiled in Table 1.

Figure 2 shows the geometry of considered system with the boundary and initial conditions. Point $A$ indicates the position of the fixed point for the casting and the mold. It provides appropriate positioning of the regions and also makes possible their free deformations. Additionally artificial stiffness $k_{s}$ is added to the coefficients on the main diagonals of $\mathbf{K}_{D M}$ and $\mathbf{K}_{D C}$. It makes the stiffness matrices well-conditioned. Horizontal dashed line at $y=0.2 \mathrm{~m}$ is the place where the temperature distribution is measured. Discontinuous temperature distribution as an effect of the thermal resistance of the air gap is clearly visible in the Figure 3 . The local width of the gap at this moment at point $B$ is $0.6 \mathrm{~mm}$. In Figure 4 the disconnected finite element meshes are shown in the neighborhood of point $B$. The air gap between the casting and the mold is present.
Table 1.

Material properties of the casting and the mold [9]

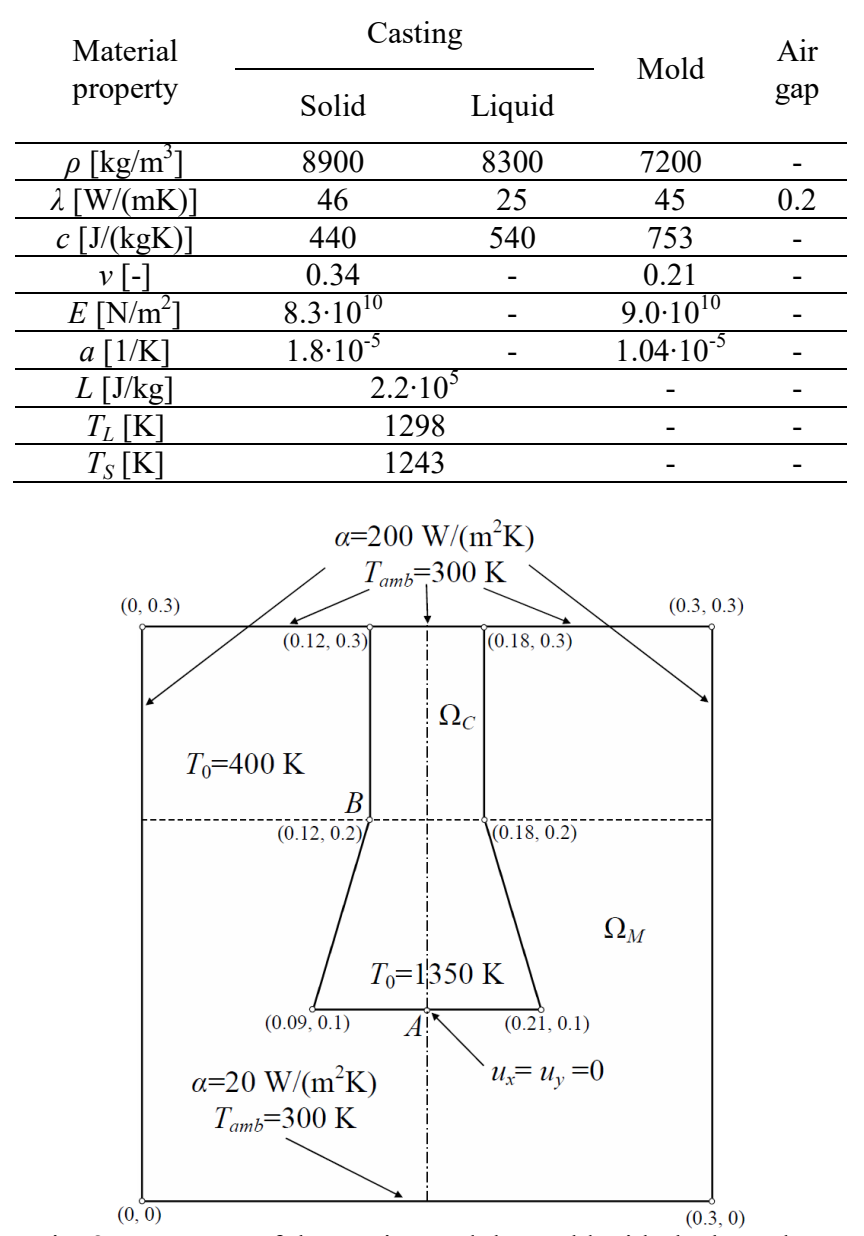

Fig. 2. Geometry of the casting and the mold with the boundary and initial conditions

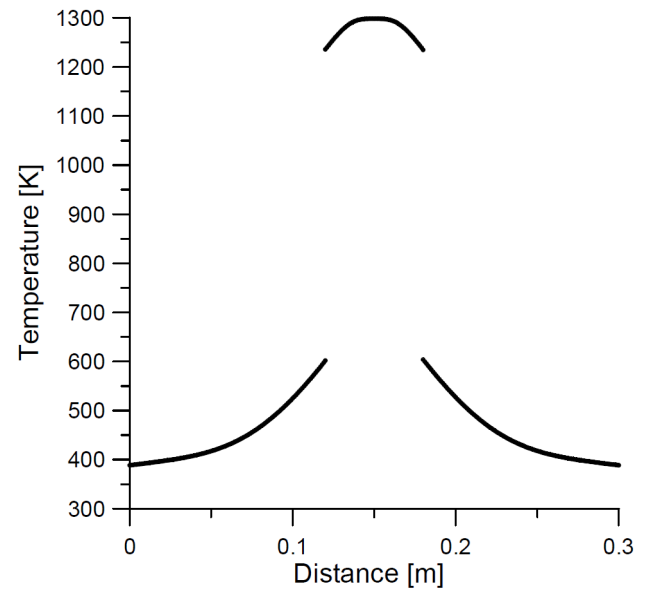

Fig. 3. Horizontal temperature distribution $(y=0.2 \mathrm{~m})$ at $t=100 \mathrm{~s}$ 


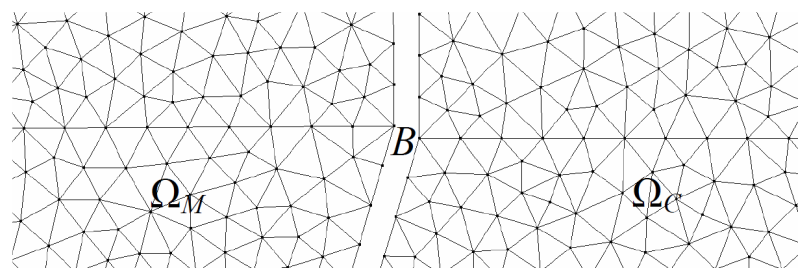

Fig. 4. Air gap in the vicinity of point $B$ at $t=100 \mathrm{~s}$

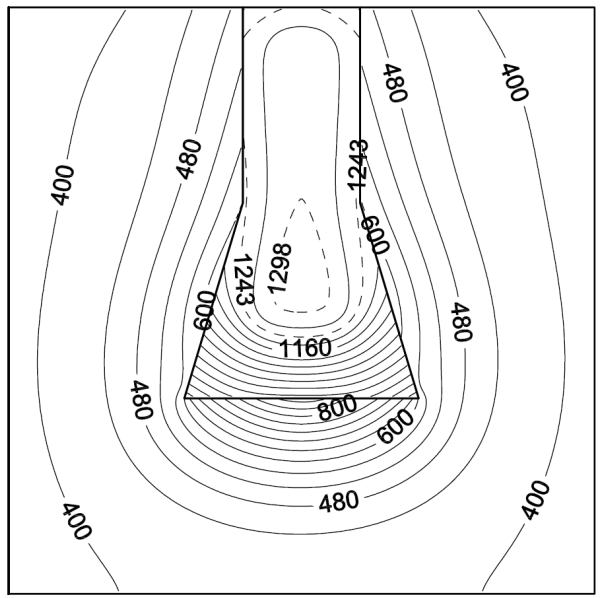

Fig. 5. Temperature distribution in the casting and the mold at $t=100 \mathrm{~s}$ (unit: K)

Figure 5 shows instantaneous temperature distribution in the considered system. Thermal resistance introduced by the air gap influences the distribution of the isothermes. The region of the mold is significantly colder than the casting and the boundary between them is the area of discontinuity of temperature except the bottom where is almost ideal contact. The solidification process ended after $350 \mathrm{~s}$

\section{Conclusions}

Described approach based on the independent spatial discretization of the mold and the casting saves significant amount of operational memory because of sequential solving of the global set of equations. Presented model makes possible to take into account the important effect of air gap on the rate of solidification process. The main purpose of the further work is the introduction of mechanical interactions between thermally deformed regions to the model. It is crucial to track the evolution of the air gap in the case of the complex external boundaries of the casting.

\section{References}

[1] Cooper, M.G., Mikic, B.B. \& Yovanovich, M.M. (1969). Thermal contact conductance. International Journal of Heat and Mass Transfer. 12(3), 279-300. DOI: 10.1016/00179310(69)90011-8.

[2] Longa, W. (1973). Solidification of castings in sand molds. Katowice: Wyd. Sląsk. (in Polish).

[3] Schneider, G.E., Strong, A.B. \& Yovanovich, M.M. (1977). Transient thermal response of two bodies communicating through a small circular contact area. International Journal of Heat and Mass Transfer. 20(4), 301-308. DOI: 10.1016/0017-9310(77)90150-8.

[4] Nishida, Y., Droste, W. \& Engler, S. (1986). The air-gap formation process at the casting-mold interface and the heat transfer mechanism through the gap. Metallurgical and Materials Transactions B. 17(4), 833-844. DOI: 10.1007/BF02657147.

[5] Majchrzak, E., Mendakiewicz, J. \& Piasecka-Belkhayat, A. (2005). Algorithm of the mould thermal parameters identification in the system casting-mould-environment. Journal of Materials Processing Technology. 164-165, 15441549. DOI: 10.1016/j.jmatprotec.2005.02.021

[6] Dyja, R., Gawrońska, E. \& Sczygiol, N. (2015). The effect of mechanical interactions between the casting and the mold on the conditions of heat dissipation: a numerical model. Archives of Metallurgy and Materials. 60(3A), 1901-1909. DOI: 10.1515/amm-2015-0324.

[7] Matlak, J. \& Słota, D. (2015). Solution of the pure metals solidification problem by involving the material shrinkage and the air-gap between material and mold. Archives of Foundry Engineering. 15(SI .3), 47-52.

[8] Luo, J., Liu, X. \& Wang, X. (2016). Analysis of temperature field, heat and fluid flow of two-phase zone continuous casting $\mathrm{Cu}-\mathrm{Sn}$ alloy wire. Archives Foundry Engineering. 16(1), 33-40. DOI: 10.1515/afe-2015-0099.

[9] Mochnacki, B., Suchy. J.S. (1993). Modeling and simulation of solidification of castings. Warszawa. (in Polish).

[10] Sowa, L. (2014). Numerical modelling of fluid flow and thermal phenomena in the tundish of CSC machine. Archives of Foundry Engineering. 14(1), 103-106.

[11] Węgrzyn-Skrzypczak, E. \& Skrzypczak, T. (2015). Modeling of thermal contact through gap with the use of Finite Element Method. Journal of Applied Mathematics and Computational Mechanics. 14(4), 145-152. DOI: 10.17512/jamcm.2015.4.15. 